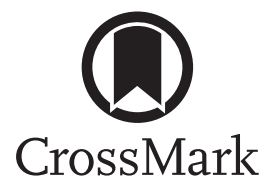

\section{Xpert MTB/RIF Ultra: the long-awaited game changer for tuberculous meningitis?}

To the Editor:

Mycobacterium tuberculosis (MTB) is the leading infectious cause of death globally and an estimated 10.4 million incident cases of tuberculosis (TB) and 1.8 million deaths occurred in 2015 [1]. Central nervous system TB, including tuberculous meningitis and brain tuberculomas, is challenging to diagnose and carries a high risk of severe disability and death [2]. Early diagnosis and prompt initiation of TB treatment offer the best chance of a good neurological outcome; however, an MTB culture of cerebrospinal fluid (CSF), the current reference standard for tuberculous meningitis, usually requires several weeks or longer for growth and is too slow for initial clinical decision-making. In addition, CSF microscopy using ZiehlNeelsen staining has a very low sensitivity [2] and, as such, integration of clinical and laboratory findings, as well as neuroimaging results, is necessary in the majority of cases to make a probable or possible diagnosis of tuberculous meningitis in the absence of microbiological confirmation [2,3].

Rapid advances in nucleic acid amplification technology (NAAT) and next-generation sequencing are revolutionising the diagnosis of many infectious diseases. Xpert MTB/RIF (Cepheid, Sunnyvale, USA), a fully automated point-of-care NAAT for the detection of MTB and mutations associated with rifampicin resistance, was recommended for use by the World Health Organization (WHO) in December 2010 for the diagnosis of pulmonary TB [4]. With concessional pricing, Xpert MTB/RIF is now available for use in the public sector and by non-governmental organisations in the majority of high burden developing countries [5]. Multiple studies have confirmed both the high sensitivity of Xpert MTB/RIF for detecting pulmonary $\mathrm{TB}(88 \%)$ and the strong negative predictive value (NPV) of Xpert MTB/RIF for pulmonary TB (greater than 98\%) [4]. In contrast, reported sensitivities of Xpert MTB/RIF for the detection of tuberculous meningitis vary widely and are disappointingly low (51-100\% against culture; pooled sensitivity: $80.5 \%$ and $20-86 \%$ against a composite reference standard; pooled sensitivity: 62.8\%) [6]. Pooled specificities are high (greater than 97.8\%) [6], although detection of MTB in CSF using Xpert MTB/RIF may depend on sample preparation (condition, volume, centrifugation) $[6,7]$.

In my experience, widespread access to Xpert MTB/RIF, combined with the WHO's 2013 policy recommendation for its use as the initial diagnostic test for tuberculous meningitis [4], has fostered an increasing reliance on laboratory diagnosis over clinical diagnosis for tuberculous meningitis. The high sensitivity and NPV of Xpert MTB/RIF for pulmonary TB have been assumed to be similarly high for extrapulmonary TB by clinicians unfamiliar with the published evidence to the contrary [6]. Many patients with tuberculous meningitis will have negative Xpert MTB/RIF results from CSF, an example of which is illustrated in figure 1. Furthermore, patients with brain tuberculomas without meningitis are likely to have very low bacillary levels in CSF and negative Xpert MTB/RIF results. Delaying or failing to initiate TB treatment on the basis of a negative Xpert MTB/RIF result from CSF can have serious and potentially deadly consequences. Clinicians should be familiar with the WHO Xpert MTB/RIF implementation manual [4] which states "...Individuals suspected of having extrapulmonary TB but who have had a single negative result from Xpert MTB/RIF should undergo further diagnostic testing, and those for whom there is a high clinical suspicion for TB (especially children) should be treated even if an Xpert $M T B / R I F$ result is negative or if the test is not available...". The 2017 guidelines of the Infectious Diseases Society of America, endorsed by the European Respiratory Society, recommend the use of NAAT on specimens obtained from sites of suspected extrapulmonary TB with the caution "...However, a negative NAAT test may not be used to exclude TB because false-negative results are exceedingly common...” [8].

@ERSpublications

Xpert MTB/RIF Ultra is a promising new technology to aid the diagnosis of tuberculous meningitis http://ow.ly/pwCy30eORBm

Cite this article as: Chin JH. Xpert MTB/RIF Ultra: the long-awaited game changer for tuberculous meningitis? Eur Respir J 2017; 50: 1701201 [https://doi.org/10.1183/13993003.01201-2017]. 


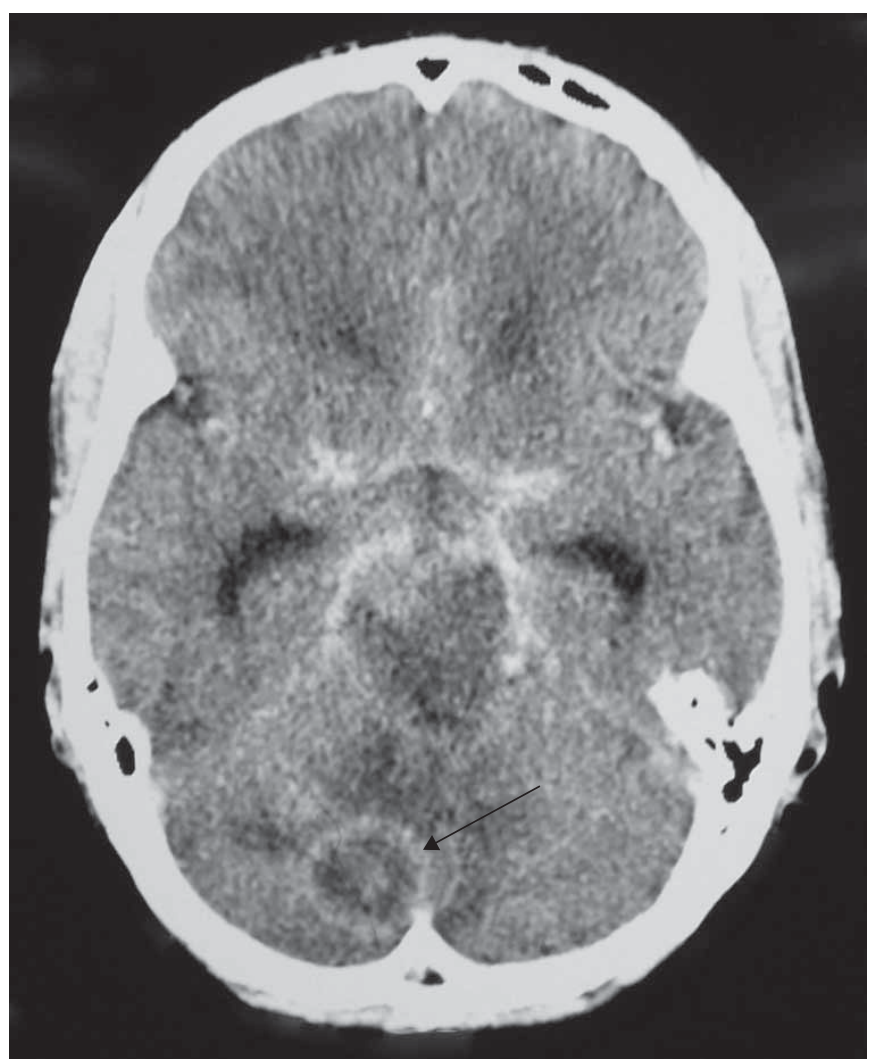

FIGURE 1 Contrast-enhanced brain computed tomoraphy image of a patient with tuberculous meningitis showing basal meningeal enhancement, enlarged lateral ventricles, and ring-enhancing lesions (arrow). Clinical diagnosis was confirmed on post mortem examination. Pre mortem Xpert MTB/RIF of cerebrospinal fluid was negative.

The Xpert MTB/RIF assay has been a game changer for the diagnosis and treatment of pulmonary TB; however, tuberculous meningitis remains a clinical diagnosis that can be confirmed but not ruled out by its use. The recent development of a next generation Xpert MTB/RIF assay, the Xpert MTB/RIF Ultra (Cepheid, Sunnyvale, USA) (Ultra), is welcome news. Ultra has an enhanced limit of detection of 15.6 bacterial colony-forming units. $\mathrm{mL}^{-1}$ (compared to 114 colony-forming units. $\mathrm{mL}^{-1}$ for Xpert MTB/RIF) [9]. The Global Tuberculosis Programme of the WHO convened a Technical Expert Consultation in January 2017 to evaluate the findings of a multi-centre non-inferiority diagnostic study comparing Ultra with Xpert MTB/RIF for the diagnosis of TB and the detection of rifampicin resistance [10]. The main study, which enrolled 1520 adults with signs and symptoms of pulmonary TB, reported a 5\% higher sensitivity but a $3.2 \%$ lower specificity of Ultra compared to Xpert MTB/RIF [9]. A single study of tuberculous meningitis conducted in Uganda, which included a new semi-quantitative "trace" category that corresponds to the lowest bacillary burden for MTB detection, demonstrated a sensitivity for detection of MTB in CSF of $95 \%$ for Ultra (21 out of 22) compared to $45 \%$ for Xpert MTB/RIF (10 out of 22) [9].

On March 24, 2017, the WHO recommended the use of Ultra as a replacement for the current Xpert MTB/ RIF cartridges in all settings. Is Ultra the long-awaited game changer for the diagnosis of tuberculous meningitis? Perhaps! Larger prospective studies are needed to determine the positive predictive values (PPVs) and NPVs of Ultra for diagnosing tuberculous meningitis. At present, Xpert MTB/RIF or Ultra testing of CSF should be performed, if available, on all suspected cases of tuberculous meningitis. The results should be interpreted in conjunction with other laboratory data, clinical information and neuroimaging results to make a diagnosis of tuberculous meningitis using experienced clinical judgement.

Jerome H. Chin $($ )

Dept of Neurology, NYU Langone Health, New York, NY, USA.

Correspondence: J.H. Chin, Dept of Neurology, NYU Langone Health, 550 1st Avenue, New York 10016, NY, USA. Email: chinj@asapp.org

Received: June 182017 | Accepted after revision: Aug 022017

Conflict of interest: None declared. 


\section{References}

1 Global tuberculosis report 2016. Geneva, World Health Organization, 2016. http://apps.who.int/iris/bitstream/ 10665/250441/1/9789241565394-eng.pdf?ua=1 Date last accessed: September 01, 2017.

2 Chin JH. Tuberculous meningitis: diagnostic and therapeutic challenges. Neurol Clin Pract 2014; 4: 199-205.

3 Marais S, Thwaites G, Schoeman JF, et al. Tuberculous meningitis: a uniform case definition for use in clinical research. Lancet Infect Dis 2010; 10: 803-812.

4 Xpert MTB/RIF implementation manual: technical and operational 'how-to': practical considerations. Geneva, World Health Organization, 2014. http://apps.who.int/iris/bitstream/10665/112469/1/9789241506700_eng.pdf? ua=1 Date last accessed: September 01, 2017.

5 Albert H, Nathavitharana RR, Isaacs C, et al. Development, roll-out and impact of Xpert MTB/RIF for tuberculosis: what lessons have we learnt and how can we do better? Eur Respir J 2016; 48: 516-525.

6 Denkinger CM, Schumacher SG, Boehme CC, et al. Xpert MTB/RIF assay for the diagnosis of extrapulmonary tuberculosis: a systematic review and meta-analysis. Eur Respir J 2014; 44: 435-446.

7 Bahr NC, Marais S, Caws M, et al. GeneXpert MTB/Rif to diagnose tuberculous meningitis: perhaps the first test but not the last. Clin Infect Dis 2016; 62: 1133-1135.

8 Lewinsohn DM, Leonard MK, LoBue PA, et al. Official American Thoracic Society/Infectious Diseases Society of America/Centers for Disease Control and Prevention clinical practice guidelines: diagnosis of tuberculosis in adults and children. Clin Infect Dis 2017; 64: 111-115.

9 A multicenter non-inferiority diagnostic accuracy study of the Ultra assay compared to the Xpert MTB/RIF assay. Geneva, FIND, 2017. www.finddx.org/wp-content/uploads/2017/03/Ultra-WHO-report_24MAR2017_FINAL.pdf Date last updated: March 24, 2017. Date last accessed: September 01, 2017.

10 WHO meeting report of a technical expert consultation: non-inferiority analysis of Xpert MTB/RIF Ultra compared to Xpert MTB/RIF. Geneva, World Health Organization, 2017. http://apps.who.int/iris/bitstream/10665/ 254792/1/WHO-HTM-TB-2017.04-eng.pdf?ua=1 Date last accessed: September 01, 2017. 